Rev Biomed 2016; 27:43-46

Semblanza Académica

\title{
Eduardo y Francisco Laviada: una sola semblanza gemelar de dos médicos
}

\author{
Hugo Antonio Laviada-Molina
}

Escuela de Ciencias de la Salud de la Universidad Marista de Mérida

Los doctores Eduardo Antonio y Francisco Antonio Laviada Arrigunaga nacieron en la ciudad de Mérida, Yucatán, el 4 de octubre de 1921. Hijos de don Antonio Laviada Arana y doña Fausta Arrigunaga, curandera empírica a decir de sus hijos, cursaron la carrera de Medicina en la Universidad de Yucatán. Antes, jóvenes inquietos, fueron promotores del movimiento Scout en Yucatán. Fueron expulsados y luego readmitidos de la Universidad siendo preparatorianos, por defender públicamente sus convicciones, en una época de turbulencia política de México.

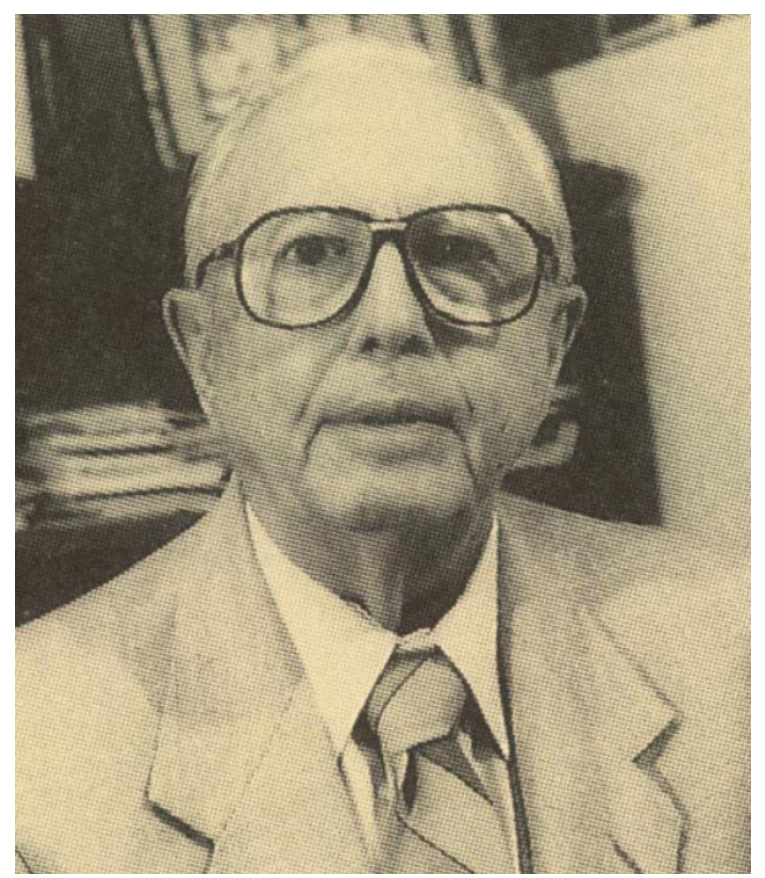

Dr. Eduardo Laviada
Se graduaron de médicos en 1949 después de haber sido internos del Hospital O’Horán y haber realizado su servicio social, Eduardo en la localidad campechana de Bécal, y Francisco en el municipio de Peto en Yucatán. Siempre se refirieron a esta época como una de las más importantes para su formación profesional. Gracias a una beca gubernamental, de 1949 a 1951, ambos realizaron estudios de posgrado en España, en el Hospital Provincial de Madrid, donde obtuviero el Doctorado en Medicina. Durante sus estudios de la especialidad en

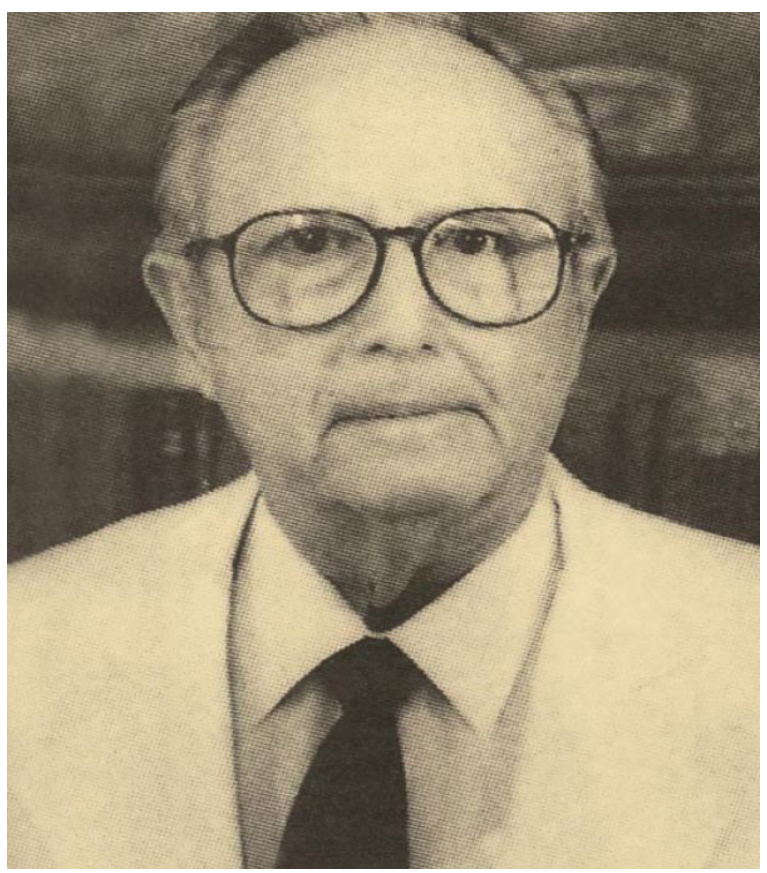

Dr. Francisco Laviada

Autor para correspondencia: Dr. Hugo Antonio Laviada Molina E-mail: halm611031@hotmail.com

Recibido: el 8 de septiembre de 2015 Aceptado para publicación: el 9 de septiembre de 2015

Este documento está disponible en http://www.revbiomed.uady.mx/pdf/rb162716.pdf

Vol. 27, No. 1, enero-abril de 2016 


\section{Laviada-Molina}

Madrid, trabajaron bajo la dirección del profesor Gregorio Marañón, su inolvidable maestro: Marañón fue un científico, historiador, escritor y pensador español, cuyas obras en los ámbitos científico e histórico tuvieron una gran relevancia internacional. Posteriormente, de 1951 a 1952, continuaron sus estudios de posgrado en el Hospital Brussais de París, Francia bajo la tutela de los profesores Degennes y Deltour.

Regresaron a México en 1953, Eduardo ya como Endocrinólogo y Francisco como Gastroenterólogo, para incorporarse de inmediato al Hospital O'Horán donde se dedicaron al ejercicio profesional y a la enseñanza de la Medicina Interna, la Gastroenterología y la Endocrinología. En este hospital Eduardo desempeñó sucesivamente los cargos de: Adjunto del Servicio de Medicina de Hombres; jefe del Servicio de Medicina de Mujeres; jefe del Servicio de Diabetología (del que fue fundador) y jefe de Enseñanza durante los 17 años comprendidos entre 1965 y 1982 en los que organizó la enseñanza de pregrado y luego la de postgrado que empezaba a formalizarse en ese entonces en nuestro Hospital-Escuela con las primeras residencias de Especialidad. Eduardo

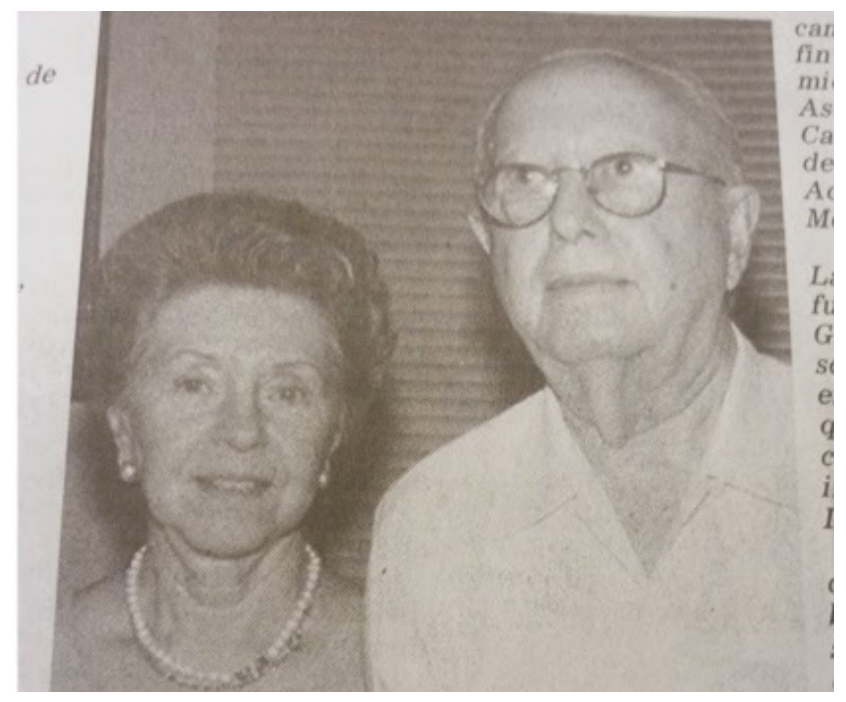

Dr. Eduardo Laviada Arrigunaga y esposa, Sra. Noemí Molina Duarte, con quien contrajo nupcias el 11 de diciembre de 1954 se casó con la señora Noemí Molina Duarte; de ese matrimonio nacieron siete hijos Noemí, Eduardo, Hugo Antonio, Jorge Antonio, Isolina, Alejandrina y Graciela.

Por otro lado, Francisco contrajo matrimonio con la señora María Elena Rodríguez y fruto de esa unión nació su hija Mary Laviada Rodríguez. Fue profesor de Infectología, Gastroenterología y Medicina Interna de muchas generaciones de médicos, y jefe del servicio de Medicina Interna. También fue investigador en el Centro de investigaciones regionales Hideyo Noguchi de la UADY. Ambos publicaron trabajos científicos en revistas nacionales e internacionales indizadas, cosa poco común en esa época sobre todo para médicos de provincia con cerca de 50 publicaciones cada uno de ellos.

Maestros por vocación, se integraron a la Facultad de Medicina en 1954 como profesores de fisiología y desde entonces realizaron una labor docente, intensa y constante, no sólo en las aulas y en el hospital, sino fuera de ellos, ilustrando con el ejemplo las cualidades que se esperan de un médico. La firmeza de sus principios combinada con su irrestricto respeto por la libertad y dignidad de los demás, unidos a su natural sentido de la justicia, así como su jovialidad y calidad humana, les granjearon la confianza, la admiración y el afecto de muchas generaciones de estudiantes de medicina en quienes supieron despertar el interés por el estudio y el amor por la carrera. A pesar de su intensa actividad profesional y docente, los doctores Laviada continuaron sus estudios con entusiasmo, interés y disciplina, ya que consideraban que "detenerse es comenzar a temer el futuro". Para ilustrar su nivel de participación, basta recordar que a los congresos anuales de la Sociedad Mexicana de Nutrición y Endocrinología, de la cual fue miembro fundador, no faltó Eduardo en una sola ocasión, desde el primero, en 1961, hasta el último, en 2002 solo semanas antes de su fallecimiento.

Para ambos, sería demasiado extenso mencionar los numerosos cursos nacionales e

\section{Revista Biomédica}




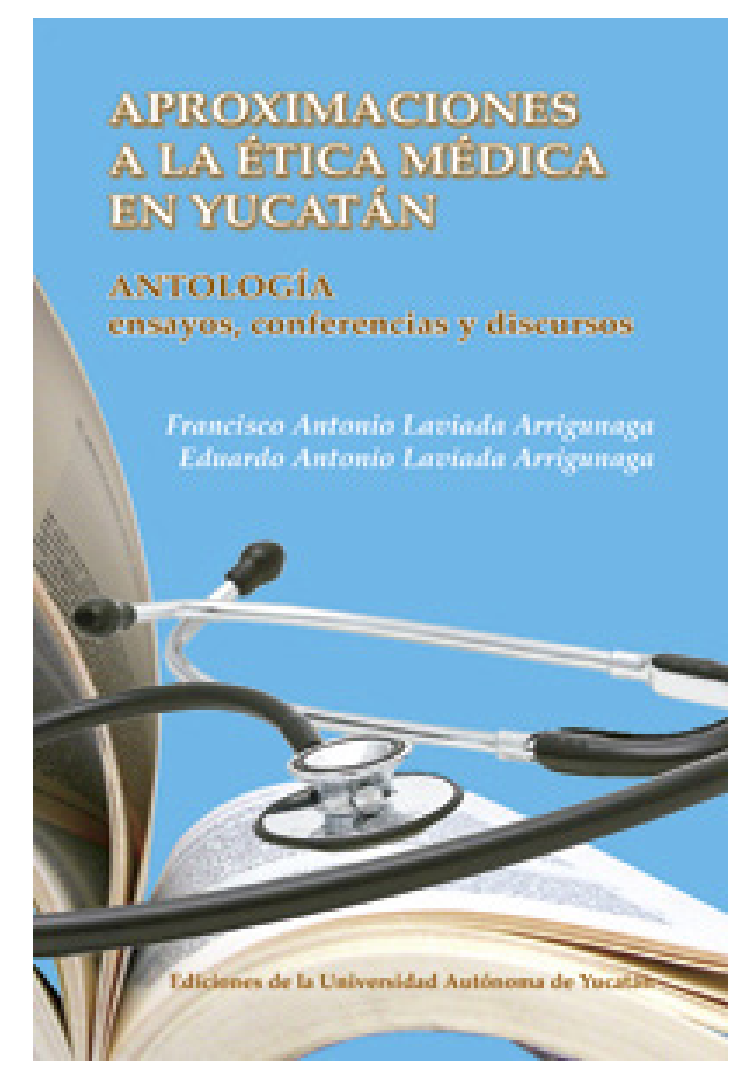

\section{Libro publicado por los hermanos Laviada}

internacionales de su especialidad y de docencia en los que participaron e impartieron, así como las muchas reuniones y congresos científicos de los que formaron parte como ponentes, expertos, coordinadores o profesores. En 1986, Eduardo fue nombrado jefe de la Unidad de Postgrado e Investigación de la Facultad de Medicina de la Universidad de Yucatán, cargo que tuvo que abandonar en septiembre de 1991 por razones de salud. Sin embargo, ambos continuaron en la Facultad por varios años más, como maestro de endocrinología y de ética médica Eduardo, y Francisco en la cátedra de Infectología. Ambos hermanos fueron también miembros titulares del American College of Physicians, de la Academia Nacional de Medicina, de la Societé de Médicine de París y de la Sociedad Médica HispanoMexicana.

Como se señaló, Eduardo fue miembro fundador de la Sociedad Mexicana de Nutrición y

\section{Semblanza de Eduardo y Franciaco Laviada}

Endocrinología, que en 1985 le otorgó el Diploma al Mérito Académico y Científico, y luego, en 1990, le distinguió con la máxima presea nacional otorgada por esta Sociedad, el Premio Nacional Salvador Zubirán. Curiosamente, ese mismo año de 1990 su hermano gemelo Francisco Antonio también recibió el reconocimiento Nacional de la Asociación Mexicana de Gastroenterología.

Eduardo fue Presidente Fundador del Capítulo Peninsular de la Sociedad Mexicana de Nutrición y Endocrinología de 1995 a 1997. Fue socio fundador de la Sociedad de Historia y Filosofía de la Medicina y miembro fundador del Patronato de la Facultad de Medicina. En el terreno de la investigación, el doctor Eduardo Laviada Arrigunaga realizó numerosos trabajos, particularmente en el área de la nutrición, el desarrollo, la diabetes y la endocrinología en general. En ellos, lo modesto de los recursos contrasta con lo interesante y lo importante de los resultados. Éstos constan en más de 50 publicaciones y artículos de revistas científicas nacionales e internacionales.

Francisco fue fundador y Presidente de la Sociedad de Medicina Interna de Yucatán. En sus trabajos de investigación, siempre procuraron la participación de los estudiantes de medicina y de médicos jóvenes, con el fin de iniciarlos en la investigación clínica, demostrándoles que las limitaciones de los recursos técnicos y económicos pueden suplirse con entusiasmo y deseos de trabajar.

En el año 2000, ambos Eduardo Antonio y Francisco Antonio recibieron la Medalla Yucatán que otorga el Gobierno del Estado a los yucatecos más destacados en todos los campos, a través del Instituto de Cultura de Yucatán.

Hasta aquí lo descrito en torno a su preparación y trayectoria personal y profesional. En una semblanza a raíz de la muerte del Dr. Eduardo, la Doctora Gloria Herrera Correa, en ese entonces Directora de la Facultad de Medicina de la Universidad Autónoma de Yucatán compartió lo siguiente: "quiero relatarles mi experiencia 


\section{Laviada-Molina}

como interna de pregrado, cuando tuve la fortuna de convivir y tenerlos como maestros. Me sentí admirada del contacto personal que establecían con sus pacientes, de la calidez y sensibilidad con la que los trataba, hablándoles en su lenguaje, utilizando términos tan comunes de nuestro pueblo maya como calhuix, chotnak, etc., lo que hacía a los pacientes reírse y sentirse confiados y atendidos, elementos tan importantes en la relación médico-paciente y fundamentales para aliviar el dolor y el sufrimiento con sólo escucharlos, lo que muchos medicamentos farmacológicos no habian podido lograr. Siempre prestos a escuchar y a responder las preguntas de sus alumnos de pregrado y posgrado, lo hacian combinando los aspectos científicos de las enfermedades con las experiencias de su vida al lado de su madre, cada uno refiriéndose a su hermano gemelo, del hospital, de sus anécdotas, de sus viajes y de sus relaciones con su familia. Fue tan fuerte la relación que establecimos como internos con ambos, Eduardo y Francisco Antonio, que toda la generación - de manera unánime-los escogimos como padrinos de generación, y ellos nos obsequiaron como regalo el siguiente ideario que voy a permitirme compartir con ustedes:

"Ideario médico que ofrecen como presente los padrinos de la generación 1979-1980 de internos de pregrado del Hospital Escuela O'Horán 1.Fomentar nuestra espiritualidad y cultura.

2. Vivir con austeridad y con la dignidad que la condición humana exige.

3. Compensarse con satisfacciones y satisfactores siguiendo una estricta escala de valores.

4. Evitar caer en el mercantilismo, conservando los honorarios a un nivel justo, por debajo del nivel esperado.
5. Olvidar negocios demasiado productivos, que por lo general no son lícitos.

6. Hacer del hogar el motivo de todos nuestros esfuerzos, sacrificios y aspiraciones.

7. Cuidar la reputación para evitar las generalizaciones que siempre hacen daño a uno mismo y al gremio médico.

8. No olvidar la preparación científica con el estudio constante y la participación en cursos de actualización, congresos, mesas redondas, etcétera.

9. Luchar sin tregua contra la adversidad, la injusticia, la corrupción y el dogmatismo.

10. Trabajar sin descanso, con plena conciencia de que el trabajo dignifica al hombre y lo libera de muchas tentaciones.

Como colofón, mencionaron "Huayo y Tony” en la última parte del discurso dirigido a aquella generación: "Tenemos fe en el futuro de la Medicina y de los jóvenes idealistas que han demostrado vocación de servicio y serán pronto nuestro relevo. No olviden la consigna de cada superarse asímismos, para mejorar el mundo".

Aunque el doctor Eduardo y su presencia física nos ha dejado, ya que falleció el 10 de febrero de 2003, su filosofía de vida, sus cualidades y valores viven en Francisco, conocido como "el Dr. Tony", el hermano gemelo que todavía nos acompaña en vida. Ambos servirán de ejemplo y orientación a futuras generaciones de estudiantes y profesionales. Y para los que tuvimos la suerte de contar con sus enseñanzas y amistad, nuestra gratitud por la labor realizada como personas, médicos y maestros, en bien de la Medicina, la enseñanza y la humanidad. Su memoria perdurará en la historia y tradición de nuestra Facultad de Medicina de Yucatán, en nuestra mente y en nuestro corazón.

\section{Revista Biomédica}

